\title{
Superconducting Magnets for a Muon Collider
}

\author{
Michael A. Green \\ Ernest Orlando Lawrence Berkeley National Laboratory \\ University of California \\ Berkeley CA. 94720
}

The existence of a muon collider will be dependent on the use of superconducting magnets. Superconducting magnets for the $\mu^{-} \mu^{+}$collider will be found in the following locations: the $\pi \pi^{+}$capture system, the muon phase rotation system, the muon cooling system, the recirculating acceleration system, the collider ring, and the collider detector system. This report describes superconducting magnets for each of these sections except the detector. In addition to superconducting magnets, superconducting RF cavities will be found in the recirculating accelerator sections and the collider ring. The use of superconducting magnets is dictated by the need for high magnetic fields in order to reduce the length of various machine components. The performance of all of the superconducting magnets will be affected the energy deposited from muon decay products.

\section{INTRODUCTION}

The proposed muon collider[1-5] consists of the following components: 1) a $10 \mathrm{GeV}$ proton source that generates about $1.5 \times 10^{15}$ protons per second, 2) a target section that produces and captures pions, 3) a section where the pions decay to muons that are phase rotated to compact bunch, 4) a muon cooling section where the muon emitance is reduced three orders of magnitude, 5) several rings to accelerate muons to $2 \mathrm{TeV}, 6$ ) the collider ring, and 7) the detector. Superconducting magnets will be found in all of these sections except for the proton source.

This report describes the superconducting solenoids in the pion capture system around the target. A brief description of the solenoids needed in the phase rotation and the muon cooling sections is presented. Dipole and quadrupoles for the recirculating accelerator rings and the collider ring are also described. This report does not include any discussion about superconducting magnets that are part of the detector system around the collider collision point.

A muon decays to two neutrinos and electron or positron (depending on the charge state of the original muon). Roughly forty percent of the muon energy ends up in the decay electron or positron. The energy in the electron or positron can be deposited in various parts of the muon collider and its subsystems. The problem of muon decay is at its worst in the collider ring, but it is a problem for superconducting magnets and RF cavities throughout the muon collider subsystems.

Muons have a life time that is dictated by its energy. The mean life of a muon at rest is about $2.197 \mu \mathrm{s}$. At the collider full energy the of $2 \mathrm{TeV}$, the muons will have a mean life of $41.6 \mathrm{~ms}$. This means that the repetition rate for the muon collider must be of the order of the muon life time in the collider ring if high colliding ring luminocities are to be maintained. In order to maintain a luminocity of $10^{35} \mathrm{~cm}^{-2} \mathrm{~s}^{-1}$ in a collider ring with a $\int *$ of $3 \mathrm{~mm}$ at the collision point, over $1.2 \times 10^{14}$ muons per second must be created and stored in a ring with $7 \mathrm{~T}$ bending magnets. The collider ring must have two bunches (one of each charge state) of $2 \times 10^{12}$ muons per bunch at a repetition rate of $30 \mathrm{~Hz}$ in order to have the desired luminocity at the collision point.

* This work was performed with the support of the Office of High Energy and Nuclear Physics, United States Department of Energy under contract number DE-AC03-76SF00098. 


\section{CAPTURE AND DECAY SOLENOIDS}

Pion capture solenoids with capture central inductions of 28 tesla and 22 tesla are described in this report Both of these capture solenoids are hybrid magnets with water cooled Bitter type solenoids inside of superconducting solenoids. The length of the capture solenoid system is determined by the type of target used to produce the pions. The capture solenoids described here are assumed to have a low Z (beryllium or carbon) targets within them.

The superconducting solenoids are graded. The outer coils are low field niobium titanium coils; the inner coils that operate at high field would be made from niobium tin. Both of the superconducting capture solenoid coils would be cooled to $1.8 \mathrm{~K}$ by a refrigerator. Both versions of the capture solenoid magnet system include a transition section where the central induction is reduced from the capture induction to the decay channel and phase rotation channel induction of 7.0 tesla. The warm bore diameter is $300 \mathrm{~mm}$ at 7 tesla in both options. The final three meters of the capture solenoid is the start of the 7 tesla muon decay channel.
The water cooled bitter solenoid absorbs $2 \mathrm{MW}$ of the $5 \mathrm{MW}$ beam energy deposited on the target at the center of the capture solenoid system. The thickness of the water cooled bitter magnet must be at least $225 \mathrm{~mm}$ so that little of the proton beam energy ends up in the $1.8 \mathrm{~K}$ superconducting solenoids Downstream from the capture solenoid, the inner wall must be heavy and water cooled in order to reduce the energy from the target that is deposited in the $7 \mathrm{~T}$ magnets. Table 1 compares the two capture solenoid designs that have been studied.

The superconducting coils for the 28 tesla capture solenoid are based on the cable in conduit superconducting coils that the National High Magnetic Field Laboratory[6] is using for their 45 tesla hybrid magnet system. The water cooled Bitter magnet is longer and has a larger bore diameter than the Florida State magnet system. The projected power numbers in Table 1 reflect the longer water cooled solenoid length. A magnet inner bore of 150 $\mathrm{mm}$ used for the 28 tesla capture solenoid will capture pions with a transverse momentum of 314 $\mathrm{MeV} / \mathrm{c}$. Figure 1 shows a cross-section of the 28 tesla solenoid.

TABLE 1 Parameters for High Field and Low Field Capture Solenoids

\section{Parameter}

Total Length of Solenoid System (mm)

Cryostat Outer Diameter (mm)

Niobium Titanium Capture Solenoid OD (mm)

Niobium Titanium Capture Solenoid Length ( $\mathrm{mm}$ )

Niobium Tin Capture Solenoid OD (mm)

Niobium Tin Capture Solenoid Length (mm)

Water Cooled Bitter Coil OD (mm)

Water Cooled Bitter Coil Bore Diameter (mm)

Water Cooled Bitter Coil Length (mm)

Decay Channel Diameter (mm)

Transition Region Length ( $\mathrm{mm}$ )

Decay Channel Length ( $\mathrm{mm})$

Capture Solenoid Total Central Induction (T)

Superconducting Solenoid Central Induction (T)

Water Cooled Solenoid Central Induction (T)

Decay Solenoid Central Induction (T)

Total Magnet System Stored Energy (MJ)

Superconducting Magnet Stored Energy (MJ)

Power Required for the Water Cooled Solenoid (MW)

310 K Cooling Required for the Magnet System* (MW)

Total Mass of the Superconducting Magnets (Metric Tons)

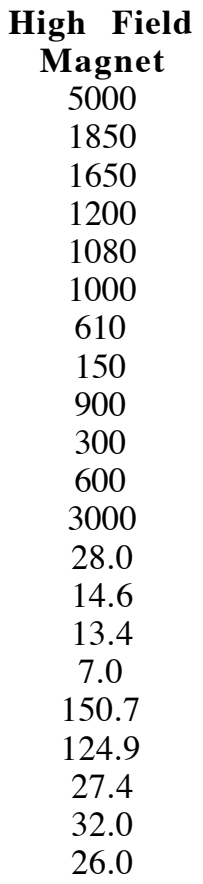

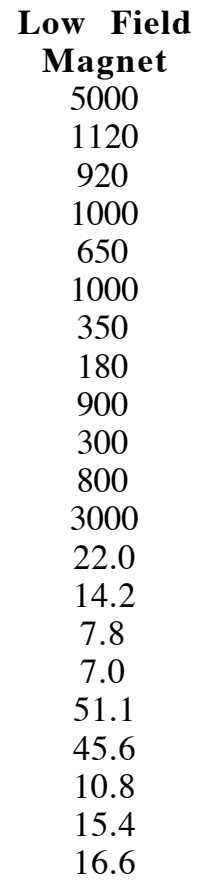

* includes the water cooling required for the beam energy into the target 


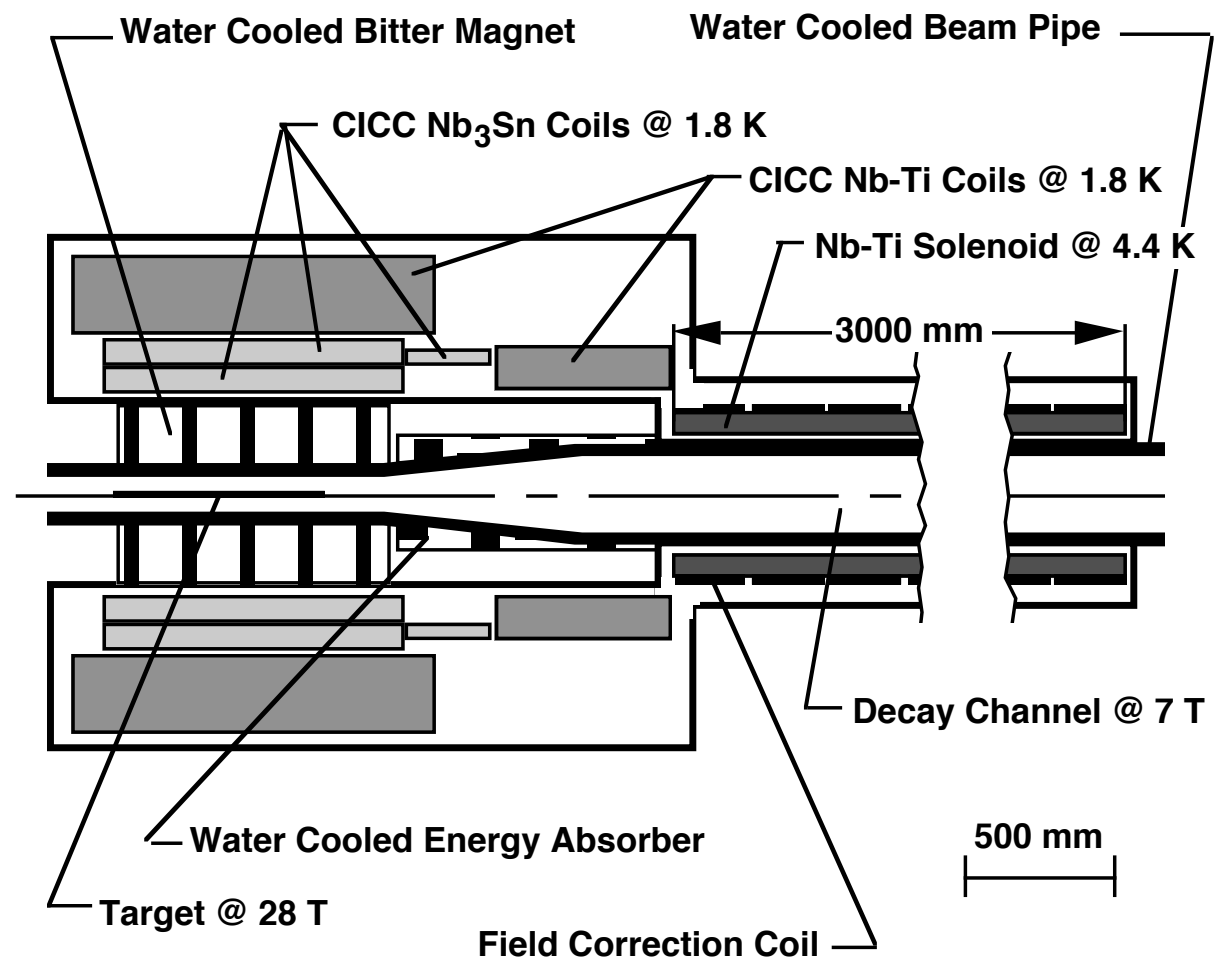

Figure 1 A Cross-section of a 28 T Hybrid Capture Solenoid using CICC Coils

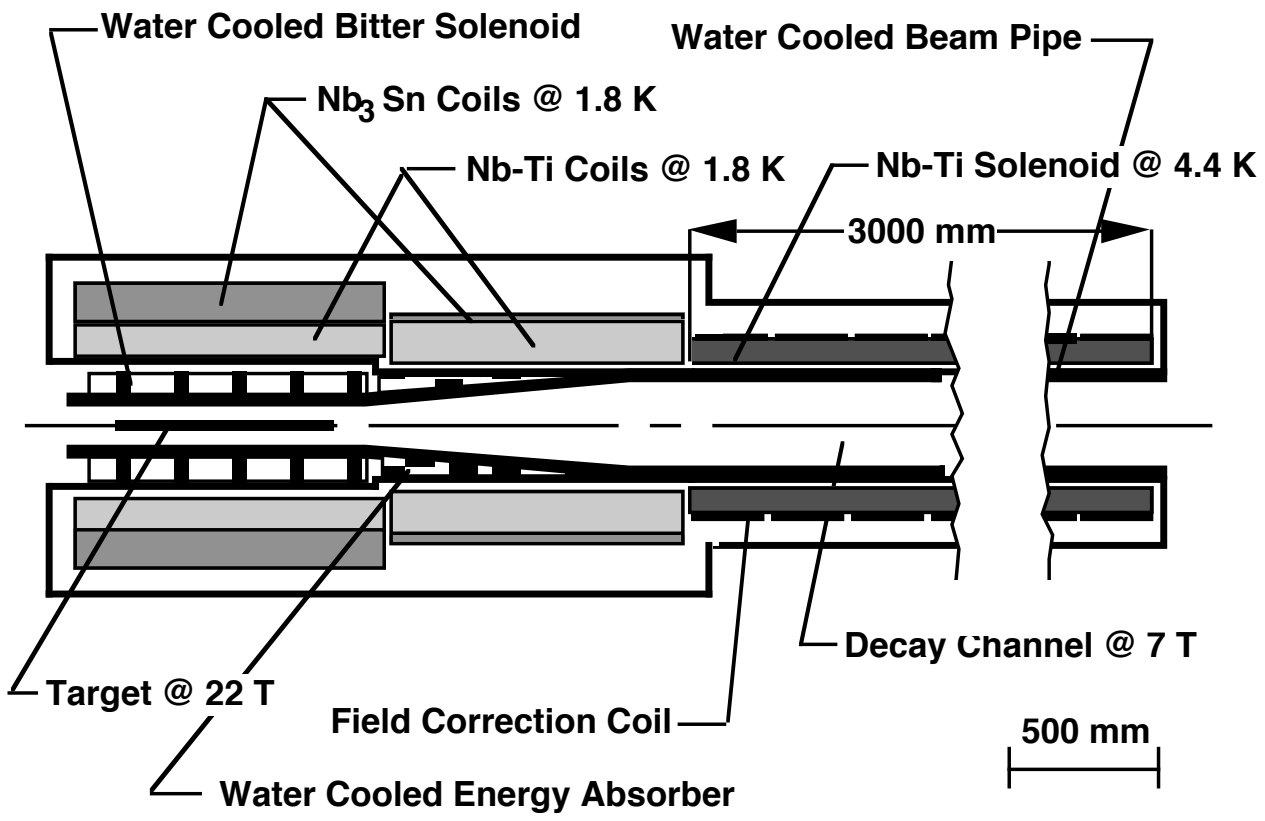

Figure 2 A Cross-section of a 22 T Hybrid Capture Solenoid using Bath Cooled Coils 
The superconducting coils for the 22 tesla capture solenoid are based on the bath cooled superconducting coils that the Francis Bitter Magnet Laboratory[7] is using for their 35 tesla hybrid magnet system. The water cooled Bitter magnet is longer and has a larger bore diameter than the MIT magnet system. The projected power numbers in Table 1 reflect this. A magnet inner bore of 180 $\mathrm{mm}$ was used for the 22 tesla capture solenoid. This magnet will capture pions with a transverse momentum of $296 \mathrm{MeV} / \mathrm{c}$. (See Figure 2 for a crosssection view.)

The theoretical capture efficiency of the 22 tesla solenoid is somewhat lower than the capture efficiency of the 28 tesla solenoid. In either case, there are problems with the water cooled Bitter solenoid section. Bitter solenoids typically have a life time that is limited by a combination of high current densities fatigue, and corrosion. The high radiation environment only makes matters worse.

The length of the decay channel between the capture solenoid and its transition section to 7 tesla is determined by the type of system used to phase rotate the pions and muons coming from the target. If a system using RF cavities is used for phase rotation, it is desirable that the RF cavities be located as close to the target as possible. The decay of the pions to muons would occur simultaneously with phase rotation. If an induction linac is used for phase rotation, a 150 meter long muon decay channel is desirable in order for the muon beam to be spread out over a length of about 30 meters. The number of muons in the channel will reach its peak about 150 meter down stream from the target.
A 150 meter long decay channel could be built from ten 15 meter long 7 tesla solenoid sections that have a warm bore of $300 \mathrm{~mm}$. Table 2 shows the basic parameters of a 150 meter long decay solenoid magnet system. The length of each magnet in the section is dictated by the quench parameters of the magnets. For 15 meter long magnet sections, the nominal superconductor plus matrix current density should be about 75 amperes per square millimeter. The warm bore tube wall thickness (assumed to be water cooled copper) is set at $25 \mathrm{~mm}$. Figure 3 shows a schematic cross-section of a decay solenoid unit.

TABLE 2 Pion Decay Solenoid Parameters

\section{Parameter}

Length of Channel (m)

Number of Units

Length per Unit (m)

Solenoid Cryostat OD $(\mathrm{mm}) \quad \sim 700$

$\mathrm{Nb}-\mathrm{Ti}$ Coil OD (mm) 530

$\mathrm{Nb}-\mathrm{Ti}$ Coil ID (mm) 380

Warm Bore Diameter (mm) 300

Bore tube Thickness (mm) 25.4

Central Induction (T) 7.0

Operating Temperature (K) $\quad \sim 4.4$

Cold Mass per Unit (metric tons) 17.8

Total Mass per Unit (metric tons) 23.0

Stored Energy per Unit* (MJ) 47.5

* at the design central induction

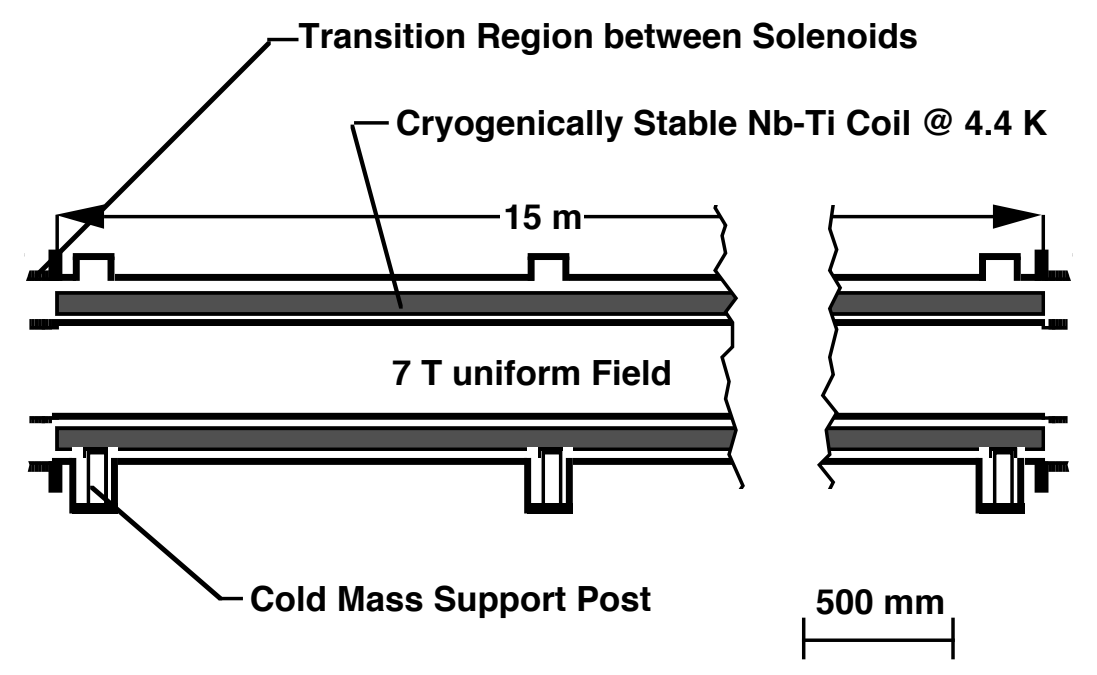

Figure 3 A Cross-section of a 7 T Decay Solenoid Section 


\section{PHASE ROTATION AND COOLING SYSTEM SOLENOIDS}

The phase rotation magnets are string of solenoids within the RF cavities or induction linac sections. The solenoids in the string have the same polarity and they occupy at least 60 percent of the space in the string. The acceleration section gap and cryostat walls occupy the space between the coils. The coils should be designed so that the solenoidal field along the string is as uniform as possible. The average induction along the string of solenoids is the same as the induction in the muon decay channel.

When RF cavities are used for phase rotation, the phase rotation must start as soon as possible downstream from the capture solenoid. Close to the target and capture solenoid, particle flux to the walls may be a factor in the design of the superconducting magnets in the RF cavity. The length of the RF cavity phase rotation system is estimated to be somewhere near 100 meters. A schematic of the RF cavities and their superconducting magnets is shown in Figure 4. It is estimated that about 80 of these cavities would be required for phase rotation provided the phase rotation starts soon enough after the pion capture.

When an induction linac is used for phase rotation, it is desirable to have a decay drift of 100 meters or more before phase rotation is started. An induction linac phase rotation system might be 250 to 300 meters long. A couple of hundred solenoids might be required for the induction linac. Like the RF phase rotation system, the uniformity of the solenoidal field down the bore of the induction linac is of some concern. A longer solenoid length with respect to the cavity length means greater the field uniformity in the phase rotation channel.

If $\mathrm{RF}$ cavities are used for phase rotation, saturation of ferromagnetic materials is not an issue. If an induction linac is used for phase rotation, the magnetic flux outside of the solenoid will be concentrated within the ferrite material that is essential to the operation of the induction linac. The effect of the magnetic field on the operation of the induction linac is not known at this time.

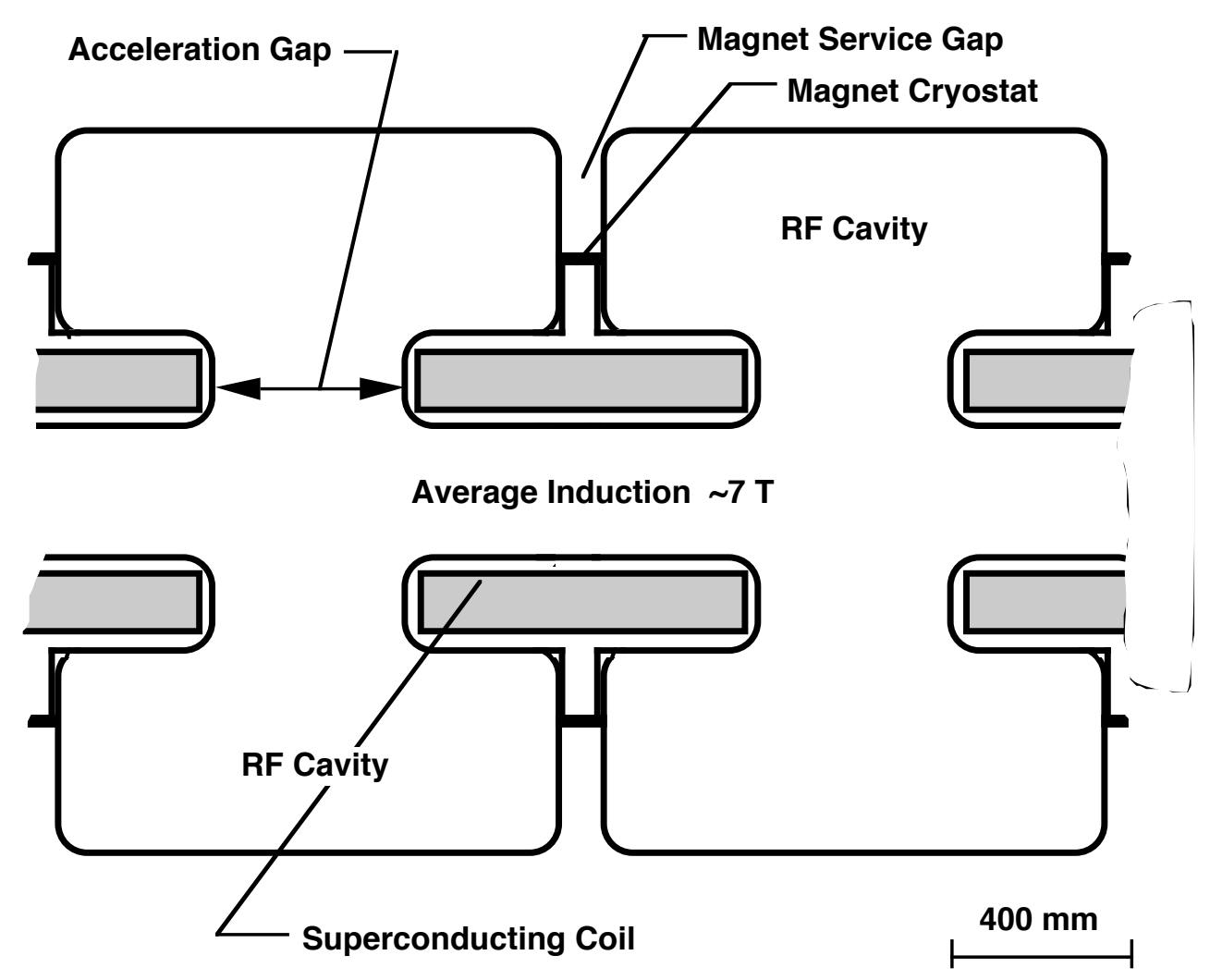

Figure 4 A Schematic View of Superconducting Solenoids within a Phase Rotation RF Cavity System 
The solenoids in the muon cooling system are very different form those in the phase rotation system. The muon cooling system uses a FOFO structure. The solenoids alternate in polarity. The cooling system solenoids start at the same central induction (at their center) as the muon decay and phase rotation system. Between the solenoid is the lithium, beryllium or lithium hydride that reduces the muon momentum (in all directions). When cooling is finished in a section, the muon are accelerated back up to about $1 \mathrm{GeV}$ (Longitudinal momentum is increased but transverse momentum is not increased as much.) As one moves down the cooler, the strength of the solenoids increases and their bores and spacings become smaller.

At the start of the cooling channel, the solenoid clear bore is about $600 \mathrm{~mm}$. At the end of the channel the clear bore drops to about $40 \mathrm{~mm}$. The central induction of the solenoids at the end of the string might be as high as 14 tesla. Figure 5 shows the cooling solenoids at the high emittance (low field) end. The cooling solenoids have alternating polarity. A radial component of field must squeeze out between the solenoid coils. The peak field in the coils can be much higher than the field along the string of solenoids. The magnetic forces on the coils want to push them apart. One can attach the coils together with a cold support, but there must be some radial access to the cooling point.

\section{ACCELERATION SECTION AND COLLIDER RING DIPOLES AND QUADRUPOLES}

The system for accelerating the muons from the cooler at an energy of $0.2 \mathrm{GeV}$ to a final energy of 2 $\mathrm{TeV}$ is assumed to contain the following components: 1) a linac to accelerate the muons from 0.2 to $2 \mathrm{GeV}, 2)$ A nine turn recirculation ring with two superconducting linacs in the straight sections to accelerate the muons from $2 \mathrm{GeV}$ to $20 \mathrm{GeV}, 3$ ) an eighteen turn recirculation ring with two superconducting linacs in the straight sections to accelerate the muons from $20 \mathrm{GeV}$ to $200 \mathrm{GeV}$, and 4) an eighteen turn recirculation ring with two superconducting linacs in the straight sections to accelerate the muons from $200 \mathrm{GeV}$ to their final energy of $2 \mathrm{TeV}$.

The recirculation rings will be separated function rings that can have up to 18 separate bores. The superconducting dipoles and quadrupoles in the bores will share a common cold iron flux return system.

The collider will be a single separated function ring of superconducting magnets that carries both the negative and the positive muons. The $\int *$ at the collision point must be about $3 \mathrm{~mm}$ in order for the desired design luminosity of $10^{35} \mathrm{~cm}^{-2} \mathrm{~s}^{-1}$ to be achieved.

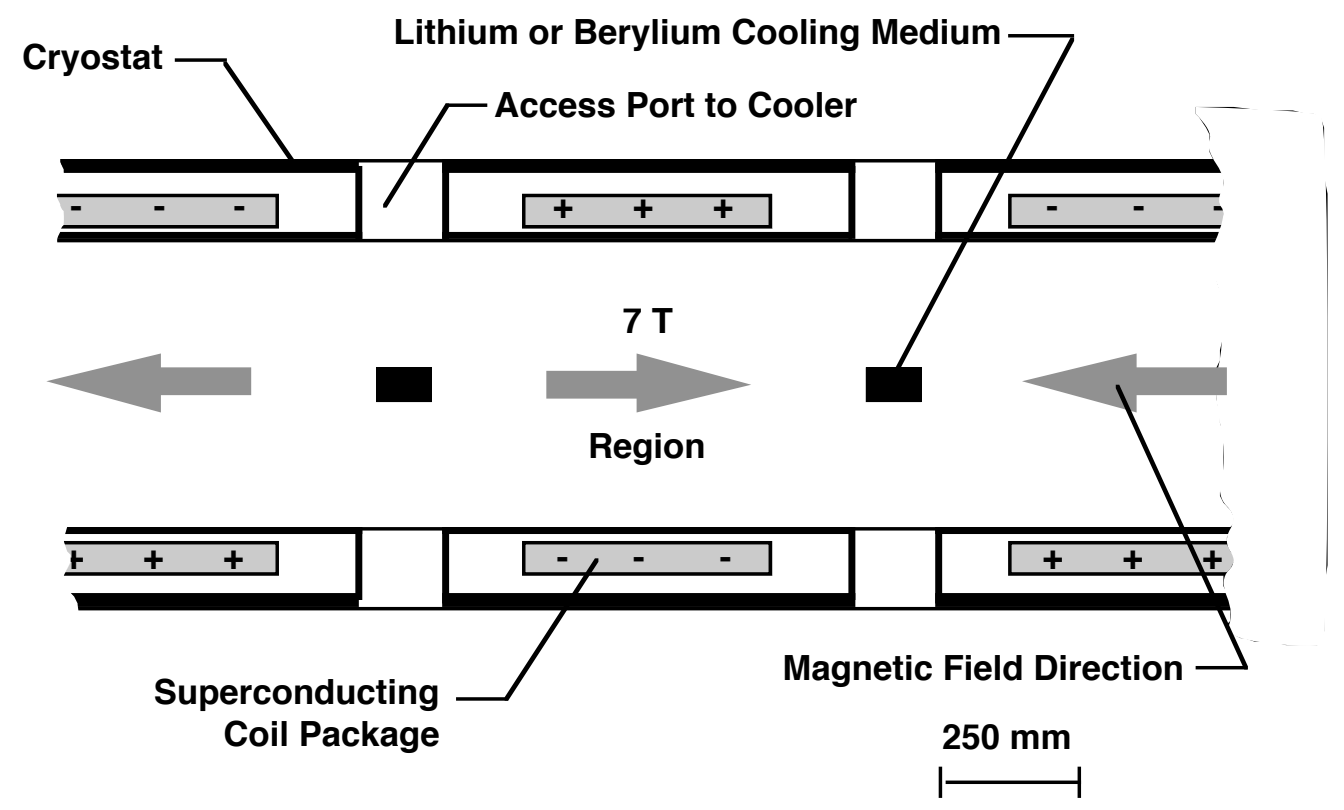

Figure 5 A Schematic Representation of Solenoids for the Muon Cooling System at the Warm End 
The number of muons that will decay in a given length $\mathrm{L}_{\mathrm{T}}$ can be estimated using the following expression:

$\mathrm{N}_{\mathrm{d}}=\frac{\mathrm{N} " \mathrm{~L}_{\mathrm{T}} \mathrm{E}_{\mathrm{O}}}{\dagger_{\mathrm{o}} "\left(\mathrm{E}_{\mathrm{T}}{ }^{\prime}+" \mathrm{E}_{\mathrm{O}}\right) " \mathrm{c}}$

where $\mathrm{N}$ is the number of muons transported through a structure per second: $\mathrm{N}_{\mathrm{d}}$ is the number of muon that decay in the structure per second; $\mathrm{L}_{\mathrm{T}}$ is the length of the structure; $\mathrm{E}_{\mathrm{T}}$ is the muon energy; $\mathrm{E}_{\mathrm{O}}$ is the muon rest mass $\left(\mathrm{E}_{\mathrm{O}}=105.7 \mathrm{MeV}\right)$; $\mathrm{c}$ is the velocity of light $\left(\mathrm{c}=2.998 \times 10^{8} \mathrm{~m} \mathrm{~s}^{-1}\right)$ : and $\dagger_{\mathrm{o}}$ is the muon decay time constant at rest $\left(\dagger_{\mathrm{o}}=2.197 \mathrm{x}\right.$ $\left.10^{-6} \mathrm{~s}\right)$. Equation 1 is applicable when the transit time for the muon through length $\mathrm{L}$ is less than the decay time constant of the muon at energy $\mathrm{E}_{\mathrm{T}}$.

The power available for deposition into the structure from the muon decay can be estimated using the following expression:

$\mathrm{P}=\sim 0.4 \mathrm{~N}_{\mathrm{d}} \mathrm{E}_{\text {ave }} \mathrm{e}$

where $\mathrm{N}_{\mathrm{d}}$ is number of muon that decay per second (See Equation 1); $E_{\text {ave }}$ is the average energy of the muon in the structure; e is the unit charge for the electron (muon have the same charge as an electron. $\mathrm{e}=1.602 \times 10^{-19} \mathrm{C} \mathrm{s}^{-1}$ ). The factor 0.4 at the start of Equation 2 represents the assumed portion of the muon energy that ends up in the decay electrons or positrons. The remainder of the muon energy is transported to the universe by the decay neutrinos.

Table 3 presents calculations for muon decay in each of the accelerator components and the collider ring. Included in the Table 3 is the number of turns through the component and the total transit length $\mathrm{L}_{\mathrm{T}}$ through the structure. Table 3 gives an estimate of the decayed muon power that is transferred to electrons and positrons. This is the portion of the decayed muon power that can end up in the superconducting magnet system. The beam flux of $\mu^{+}$and $\mu^{-}$that enters the accelerator section is assumed to be $1.8 \times 10^{14}$ muons per second (one bunch of each type with $3 \times 10^{12}$ muons per bunch at a repetition rate of $30 \mathrm{~Hz}$ ). The peak bending induction in all of the rings is assumed to be $7 \mathrm{~T}$. (Increasing the field in the collider ring dipoles will increase the luminosity at the collision point.)

The size of the region where the decay electrons, positrons and synchrotron radiation strike the wall of the vacuum chamber is determined by the vertical emittance of the beam, the beam dispersion caused by inhomogeneities of the magnetic field, and the physical size of the muon beam (say $6 \beta$ beam size from the mid plane in the vertical direction). The field quality has a larger effect than beam emittance on the size of the fan of muon decay products from the beam. If the field within the dipole is good to 1 part in 1000, the decay product fan angle from the mid plane is about 1 milliradian.

The stay clear region for the decay products as far as the magnet coils are concerned is determined by the size of the region where the decay energy strikes the wall, plus the physical size of the magnet coil supports at the mid plane, plus the thickness of multilayer insulation between the coil mid plane support structure and the vacuum chamber, and the thickness of the vacuum chamber. For a $6 \beta$ vertical beam size of $5 \mathrm{~mm}$, the minimum separation of the coils at the mid plane, surrounding the vacuum vessel would be about $15 \mathrm{~mm}$.

\begin{tabular}{|c|c|c|c|c|c|c|}
\hline Component & $\begin{array}{c}\text { Peak } \\
\text { Energy } \\
(\text { GeV })\end{array}$ & $\begin{array}{l}\text { Number } \\
\text { of turns }\end{array}$ & $\begin{array}{c}\mathbf{L} \mathbf{T} \\
(\mathbf{k m})\end{array}$ & $\begin{array}{c}\text { Decay Rate } \\
\left(\mu \mathbf{s}^{-1}\right)\end{array}$ & $\begin{array}{c}\text { Heating } \\
\text { Power } \\
(\text { kW) }\end{array}$ & $\begin{array}{l}\text { Peak Heat } \\
\text { per unit } L \\
\left(W \mathbf{m}^{-1}\right)\end{array}$ \\
\hline Linac & 2 & -NA- & 0.12 & $1.938 \times 10^{13}$ & 1.37 & -NA- \\
\hline First Ring & 20 & 9 & 1.37 & $0.653 \times 10^{13}$ & 2.49 & 4.6 \\
\hline Second Ring & 200 & 18 & 20.13 & $1.088 \times 10^{13}$ & 31.9 & 2.5 \\
\hline Third Ring & 2000 & 18 & 201.3 & $1.011 \times 10^{13}$ & 324 & 2.5 \\
\hline Collider Ring & 2000 & $1356^{* *}$ & 9.2 & $1.331 \times 10^{14}$ & 17100 & $\sim 2400$ \\
\hline
\end{tabular}




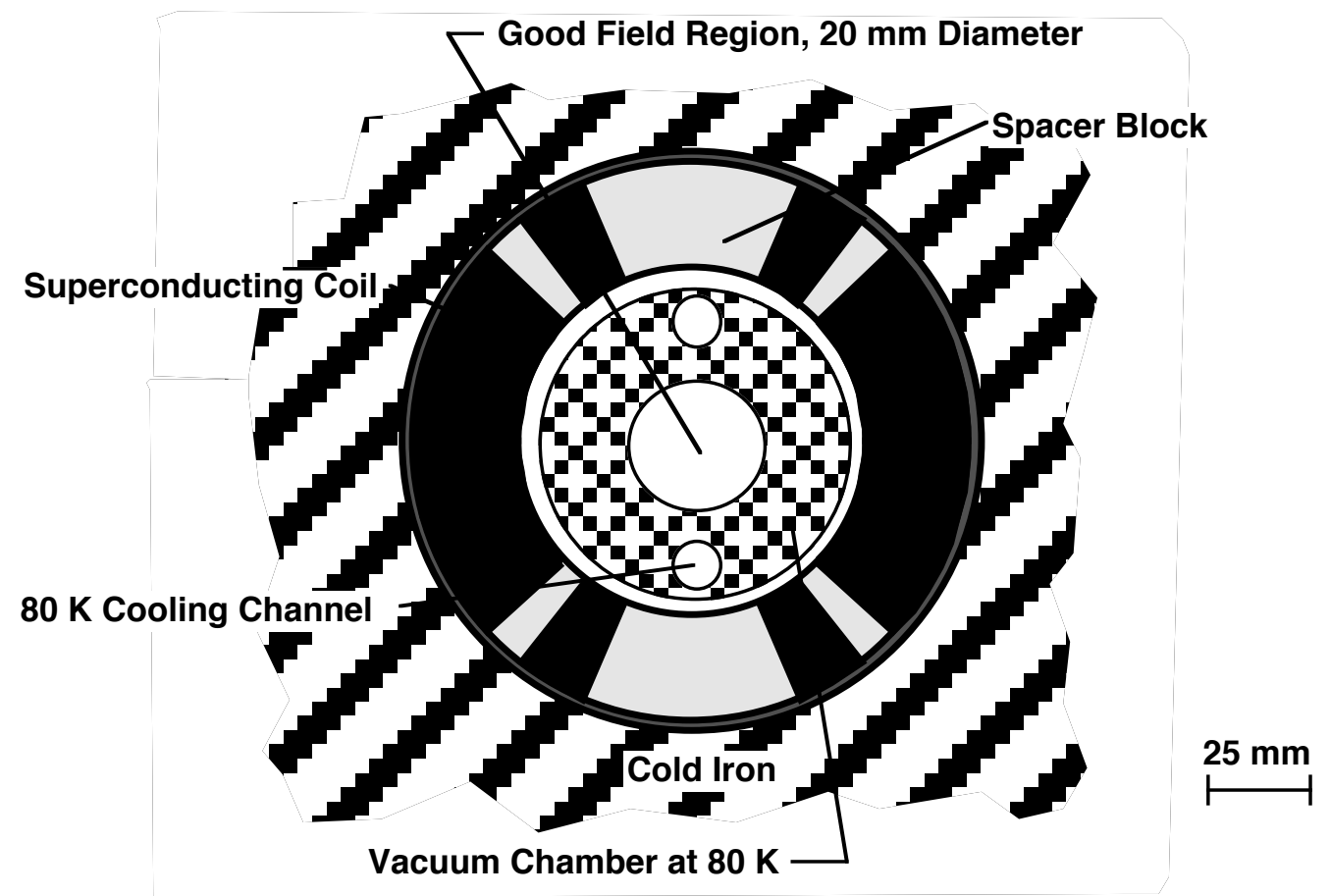

Figure 6 A Cosine Theta Dipole with a $20 \mathrm{~mm}$ Thick Vacuum Chamber Cooled to $80 \mathrm{~K}$

The design of the dipoles and quadrupoles is dependent on the percentage of the muon decay product energy that can be deposited within the $4 \mathrm{~K}$ mass of the dipole. At least three design approaches for can be considered for the superconducting dipole magnets in the various rings for the muon collider.

For the four acceleration rings a conventional cosine theta dipole design can be employed with a warm vacuum chamber that has a cooling system to remove the energy from the muon decay products. The vacuum chamber should be made from material heavy enough to reduce the energy into the $4 \mathrm{~K}$ region of the magnet by a factor of three to ten. The heavy vacuum chamber scatters the particles and reduces the energy deposition per unit volume in the superconductor. The temperature of the vacuum chamber can be as low as $40 \mathrm{~K}$. Figure 6 illustrates how a cosine theta dipole magnet can have a $20 \mathrm{~mm}$ thick metal liner at $80 \mathrm{~K}$ that absorbs energy from the decay electrons and positrons. A bronze liner would absorb 75 percent of the decay product energy; a tungsten liner would absorb 90 percent of the energy. Acceleration section quadrupoles can have a liner similar to the dipole's liner.

For the collider ring, one can use a conventional cosine theta type of dipole provided the heavy (tungsten) vacuum chamber is thick enough to reduce the energy into the superconducting coils about three orders of magnitude. The collider dipole warm bore is about $20 \mathrm{~mm}$. The thickness of tungsten needed to reduce the heating from muon decay three orders of magnitude is about $65 \mathrm{~mm}$. The cold bore of the superconducting dipole coils has to be about $160 \mathrm{~mm}$ for a magnet with a $20 \mathrm{~mm}$ bore in the liner. The heavy vacuum tube must be cooled with a fluid at room temperature. A collider ring dipole with a tungsten liner is illustrated in Figure 7 . The energy from the decay products that remains is well scattered into the coils.

Another approach to building collider ring dipole magnets is to have the coils that are completely separated on the mid plane. The iron return yoke is at room temperature. The forces pulling the coil together across the mid-plane must be carried by 300 to $4 \mathrm{~K}$ supports. (Cold iron designs might be possible and are being studied.) The coils must be separated so that less than 0.1 percent of the energy from the muon decay products ends up in the superconducting coils or its surrounding support structure that is at $4 \mathrm{~K}$. The rest of the muon decay product energy ends up in the water cooled vacuum chamber and water cooled iron at the mid plane. Figure 8 shows warm iron dipole with maximum separation between the coils. The dipole shown in Figure 8 has reasonably good field quality (about 4 parts in 10000 at a 10 mm radius). 


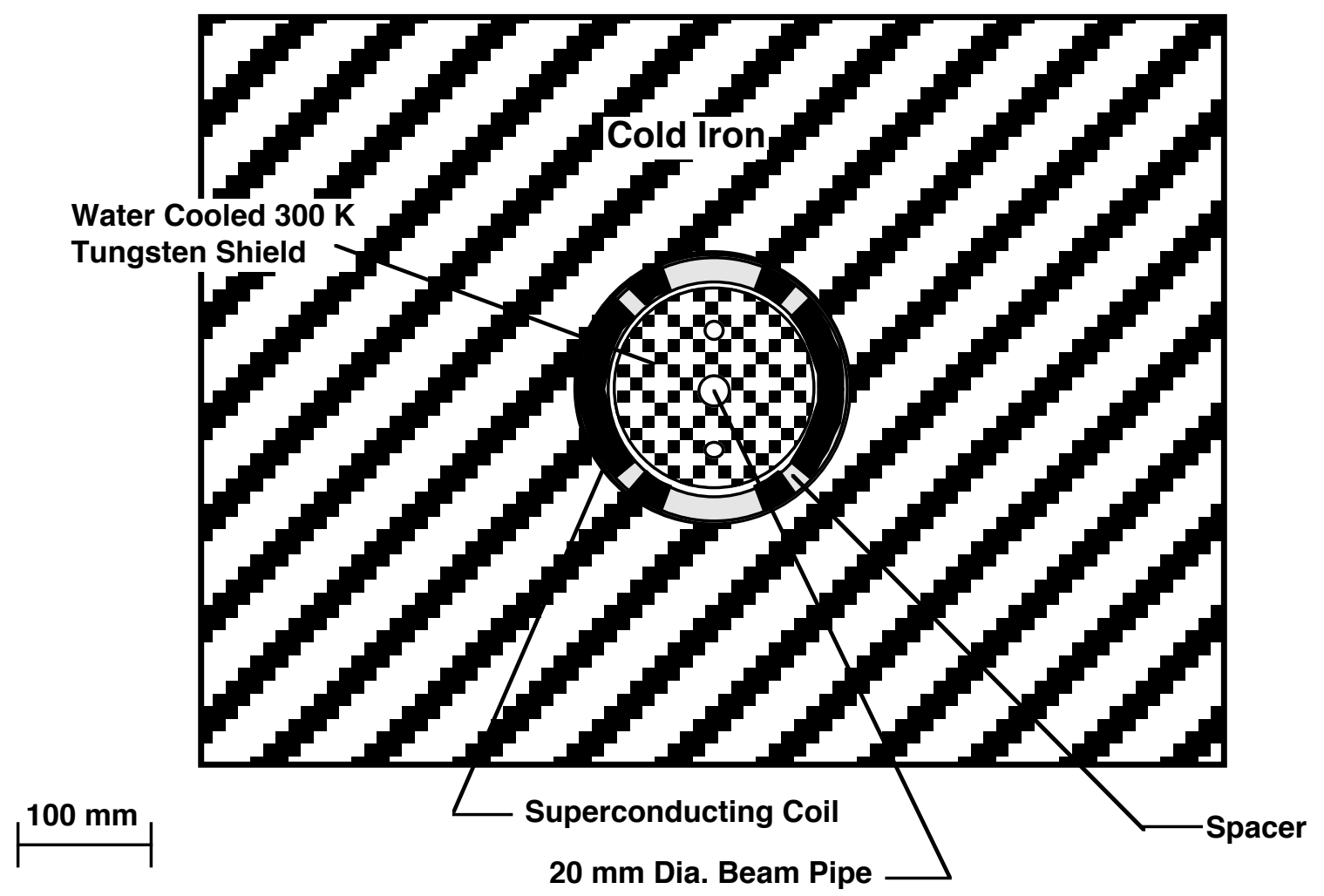

Figure 7 A Cold Iron 7 T Cosine Theta Dipole with a 65 mm Thick Tungsten Liner at $300 \mathrm{~K}$

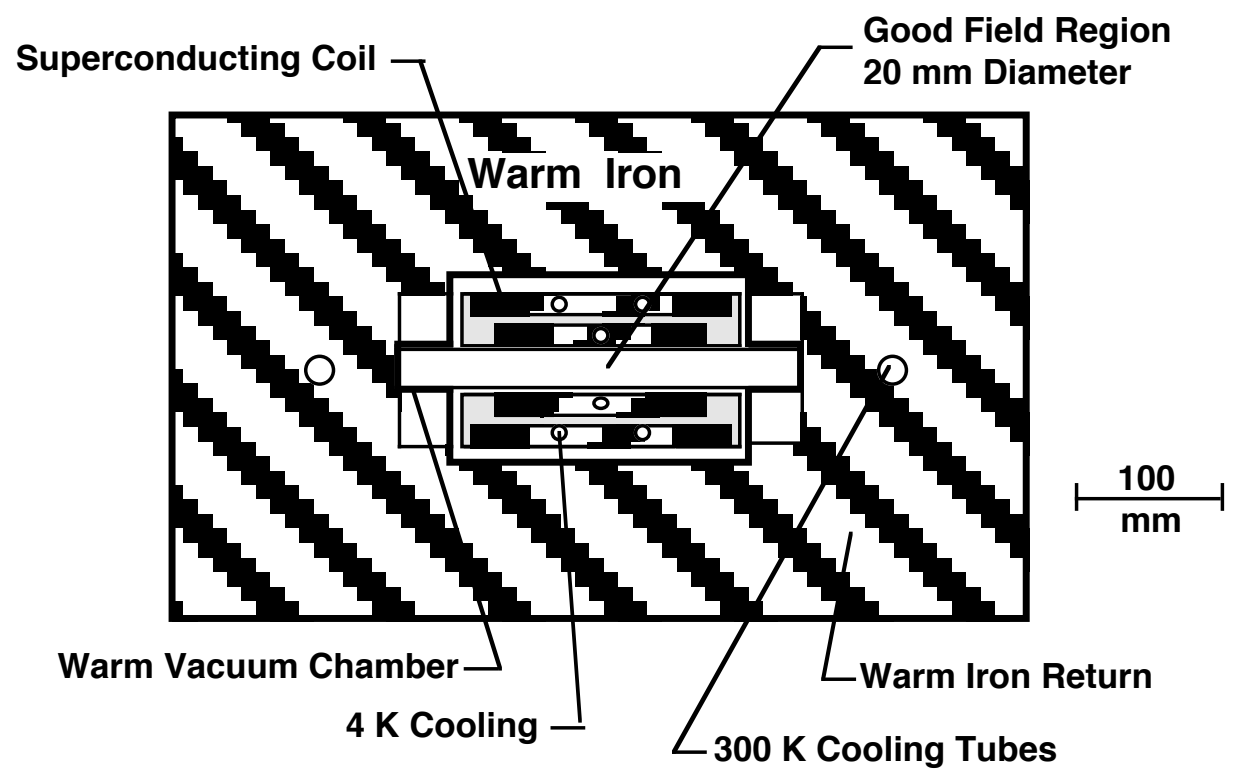

Figure 8 A 7 T Warm Iron Split Dipole that will have less than 0.1 Percent of the Muon Decay Product Power Deposited within the Superconducting Coils 
The quadrupoles will have most of the decay product energy deposited on the mid plane because the decay products from the upstream dipole will end up in the quadrupoles. Decay products produced in the quadrupoles will end up on both the horizontal and vertical axes. One problem with quadrupoles and sextupoles is that mid plane coil separation has a worse affect on field quality than it does in a dipole. A second problem with quadrupoles and sextupoles is that the coil radius has a strong effect on the gradient one can get in the bore of the magnet.

Quadrupoles in the acceleration sections of the muon collider can have a vacuum chamber similar to the dipoles, but the design gradient must be reduced. It is unattractive for the quadrupoles and sextupoles in the collider ring to be split on the mid plane. It is also very unattractive for the vacuum chamber to be $65 \mathrm{~mm}$ thick. It appears to be desirable that cell quadrupoles and sextupoles for the collider ring be conventional. For a collider ring good field aperture diameter of $20 \mathrm{~mm}$, quadrupole gradients of 100 to $120 \mathrm{~T} \mathrm{~m}^{-1}$ are possible using conventional water cooed magnets. Extra water cooling is required to absorb the energy from the decaying muons.

\section{ACKNOWLEDGMENT}

The author acknowledges the discussions he has had with Robert Palmer and Gerry Morgan of the Brookhaven National Laboratory concerning various aspects of muon collider magnet design. The author acknowledges the contribution Robert Nobel of Fermi National Laboratory concerning the design of the pion capture and the shape of the magnetic field needed for capture and transport of the pions The author acknowledges discussions he has had with H. G. Kirk and W. C. Turner concerning magnets within the RF cavities and induction linac.

This work was performed with the support of the Office of High Energy and Nuclear Physics, United States Department of Energy under contract number DE-AC03-76SF00098.

\section{REFERENCES}

1. Transparencies presented at the Second Workshop on the Physics Potential and Developmentof $\mu^{+} \mu^{-}$Colliders, Alta Mira Hotel, Sausalito California, 17-19 November 1994

2. Transparencies by R. B. Palmer from the First High Luminosity $2+2 \mathrm{TeV} \mu^{+} \mu^{-}$Collider Collaboration Meeting,, Brookhaven National Laboratory, 6-8 February 1995

3. Transparencies presented at the A Second High Luminosity $2+2 \mathrm{TeV} \mu^{+} \mu^{-}$Collider Collaboration Meeting, Fermi National Laboratory 11-13 July 1995, compiled by Robert Noble of FNL

4. Transparencies presented at the Ninth Advanced ICFA Beam Dynamics Workshop: Beam Dynamics and Technology Issues for $\mu^{+} \mu^{-}$ Colliders, Montauk, New York, 15-20 October 1995, compiled by Juan C. Gallardo of BNL

5 R. B. Palmer, A. Sessler, A. Skrinsky, A. Tollesstrup, et al., "Muon Colliders," to be published in the Proceedings of the Ninth Advanced ICFA Beam Dynamics Workshop: Beam Dynamics and Technology Issues for $\mu^{+}$ $\mu^{-}$Colliders, Montauk, New York, 15-20 October 1995, BNL-62740 (Jan. 1996)

6. Miller, J. R., Bird, M. D., Bole, S., et al, "An Overview of the 45T Hybrid Magnet System for the National High Field Magnet Laboratory," IEEE Transactions on Magnetics 30, No. 4, $\mathrm{p}$ 1563, (1994)

7. Iwasa, Y., Leupold, M. J., Weggel, R. J., and Williams J. E. C., "Hybrid III: The System, Test Results, the Next Step," IEEE Transactions on Applied Superconductivity 3, No. 1,p 58, (1993) 
SC-MAG-544

LBL-38398

UC-414

\title{
Superconducting Magnets for a Muon Collider
}

\author{
M. A. Green \\ Ernest Orlando Lawrence Berkeley National Laboratory \\ University of California \\ Berkeley CA 94720, USA
}

\section{February 1996}

* This work was performed with the support of the Office of High Energy and Nuclear Physics, United States Department of Energy under contract number DE-AC03-76SF00098. 